\title{
Depression: a characteristic comorbidity of COPD?
}

\author{
Paul W. Jones
}

Affiliations: Institute for Infection and Immunity, St George's, University of London, London, UK.

Correspondence: Paul W. Jones, Institute for Infection and Immunity, St George's, University of London, Cranmer Terrace, London SW17 ORE, UK. E-mail: pjonesdsgul.ac.uk

0

@ERSpublications

Depression is common in COPD patients and requires recognition, diagnosis and treatment http://ow.ly/z0NEG

Depression is common in the general population and is now recognised as a frequent concomitant condition in chronic obstructive pulmonary disease (COPD). However, are they truly comorbid, linked diseases? A review by HegerL and Mergl [1] in this issue of the European Respiratory Journal has appraised the available evidence and is uncertain about the conclusions to be drawn.

The authors point out that there is wide variation in the reported prevalence of depression in COPD, which may be due, in part, to a lack of standardisation of methodology, as nearly all studies of depression prevalence in COPD have used case-finding questionnaires, not formal diagnostic interviews. They argue that COPD and depression share common features such as fatigue, low physical activity, sleep disturbance and loss of interest in life, so in part, the high prevalence may be attributable to shared symptoms and inadequate diagnostic methodology. Suicide is a recognised feature of depression but it has not received wide awareness in COPD; however, they identified three studies that suggest that there is an increased risk in COPD, although that is only indirect evidence for an increased incidence of depression.

Their overall conclusion is that COPD does not appear to be a major cause of depression. Are they correct? Even if there is a link, is it a reaction to the patient's state or a systemic consequence of the disease state similar to cardiovascular disease or muscle wasting? Depression may precede the diagnosis of COPD, which they argue militates against a causal association (i.e. COPD causing depression), although that is not a compelling argument. COPD is diagnosed late in its course and there is evidence to suggest that depression may predate other conditions such as breast cancer [2]. One way of addressing this question would be to test for an association between COPD severity and level of depression. There is evidence to suggest a link between depression and exacerbations, but the mechanisms are likely to be complex [3]. It should be remembered that most definitions of an exacerbation require the patient to report to a physician, i.e. it has a behavioural component that may be affected by depression. The absence of a clear link with spirometry is not helpful either, as the relationship between increased severity of airflow limitation and increased risk of cardiovascular disease in COPD is rather weak [4]. Similarly, emphysema score may be a better predictor of osteoporosis than forced expiratory volume in $1 \mathrm{~s} \mathrm{[5].}$

Treatment for depression works but there are few trials of adequate power or quality in COPD to show convincingly whether with tricyclic agents (TCAs) or selective serotonin uptake inhibitors (SSRIs) are effective in this condition. However, there are no good grounds to assume that they will not work either, although the side-effect profiles would favour SSRIs over TCAs. There are more papers on cognitive behavioural therapy (CBT) in study populations that included patients with COPD; however, these are generally small and there is always a risk of publication bias under such circumstances. Overall they suggest that there may be benefit [6]. In some respects, this is not surprising if, as the authors of this review suggest,

Received: July 092014 | Accepted: July 092014

Conflict of interest: None declared.

Copyright @ERS 2014 
depressive symptoms in COPD are a feature of the lung disease rather than comorbid clinical depression. Elements of CBT are familiar to anyone providing a pulmonary rehabilitation programme, as it encourages the patient to examine how their actions affect how they think and feel, and thereby change their behaviour.

Where are we left after this thought-provoking review? On balance, the evidence suggests to me that there is probably an increased rate of depression in COPD on top of a high prevalence in people of similar age, and the authors point out that there is an increased risk of suicide. Diagnosis remains a problem. The UK National Institute for Health and Care Excellence guidance for adult depression [7] suggests asking two screening questions: "during the last month, have you often been bothered by feeling down, depressed or hopeless?" and "during the last month, have you often been bothered by having little interest or pleasure in doing things?". It then says that a "competent practitioner" should assess the patients and implies that person is a general practitioner. It also suggests that use of a "validated measure of symptoms, functions and/or disability" should be considered as part of the assessment. The nature of that measure is not specified but presumably it means a case-finding tool for depression, rather than a complex researchorientated depression inventory. That points to the use of an instrument such as the Hospital Anxiety and Depression Scale [8] or Center for Epidemiologic Studies Depression Scale [9]; both are available in a wide range of languages. Having made a diagnosis of clinical depression, the patient requires treatment. There are sufficient grounds to support the use of SSRIs and, where available, CBT. Use of the latter requires more research, because there are grounds for suggesting that the elements of CBT should be incorporated into the routine management of COPD patients through rehabilitation programmes. With more evidence, it is possible that CBT techniques could become part of the essential skill set of nurses and therapists involved in the care of COPD patients, whether depression is caused by COPD or is simply a concomitant condition with many shared clinical features. Either way, depression is common in COPD patients and requires recognition, diagnosis and treatment.

\section{References}

Hegerl U, Mergl R. Depression and suicidality in COPD: understandable reaction or independent disorders? Eur Respir J 2014; 44: 734-743.

2 Pössel P, Adams E, Valentine J. Depression as a risk factor for breast cancer: investigating methodological limitations in the literature. Cancer Causes Control 2012; 23: 1223-1229.

3 Laurin C, Moullec G, Bacon SL, et al. Impact of anxiety and depression on chronic obstructive pulmonary disease exacerbation risk. Am J Respir Crit Care Med 2012; 185: 918-923.

4 Mannino DM, Thorn D, Swensen A, et al. Prevalence and outcomes of diabetes, hypertension and cardiovascular disease in COPD. Eur Respir J 2008; 32: 962-969.

5 Bon J, Fuhrman CR, Weissfeld JL, et al. Radiographic emphysema predicts low bone mineral density in a tobaccoexposed cohort. Am J Respir Crit Care Med 2011; 183: 885-890.

6 Fritzsche A, Clamor A, von Leupoldt A. Effects of medical and psychological treatment of depression in patients with COPD - a review. Respir Med 2011; 105: 1422-1433.

7 National Institute for Health and Care Excellence. Depression in adults with a chronic physical health problem: treatment and management (NICE clinical guidelines, No. 91). Leicester, British Psychological Society, 2010. Zigmond AS, Snaith RP. The hospital anxiety and depression scale. Acta Psychiatr Scand 1983; 67: 361-370.

9 Radloff LS. The CES-D scale. Appl Psychol Meas 1997; 1: 385-401. 